\title{
WEAK FERROMAGNETISM OF Fe INTERCALATED FULLERIDES
}

\author{
P. Byszewski ${ }^{a, b}$, R. DiDUszKo ${ }^{a}$ AND M. BARAN ${ }^{b}$ \\ anstitute of Vacuum Technology, Długa 44/50, 00-241 Warszawa, Poland \\ ${ }^{b}$ Institute of Physics, Polish Academy of Sciences \\ Al. Lotników 32/46, 02-668 Warszawa, Poland
}

The magnetic properties of $\mathrm{C}_{60} \mathrm{Fe}_{x}$ resemble spin glass with the freezing onset above $200 \mathrm{~K}$. The unusual magnetization behavior at intermediate temperatures is ascribed to bonds reorganization.

PACS numbers: 78.20.Ls, 76.70.Hb, 78.55.Kz

\section{Introduction}

The properties of fullerides [1] can be modified in a wide range by intercalation. When intercalated by alkali metals $[2,3]$ or alkaline earth metals $[4,5]$ the fullerides can exhibit superconducting properties. In the case of doping with the organic compound TDAE $\left(\mathrm{C}_{2} \mathrm{~N}_{4}\left(\mathrm{CH}_{3}\right)_{8}\right)$, the material exhibits ferromagnetic properties [6]. In this paper the results of experiments on iron doped fullerides [7] are reported.

The molecular orbitals of the pentagons on $\mathrm{C}_{60}$ with $\pi$ electrons projecting from the carbon sites are similar to those of the hydrocarbon complexes $\mathrm{C}_{5} \mathrm{H}_{5}$. It could be expected that complexes $\mathrm{C}_{60} \mathrm{FeC}_{60}$ bound by pentagons should be formed, exhibiting properties similar to those of ferrocene $\mathrm{C}_{5} \mathrm{H}_{5} \mathrm{FeC}_{5} \mathrm{H}_{5}$. The ferrocene molecule consists of two parallel pentagonal hydrocarbon rings with $\mathrm{Fe}$ atom between them. To the iron ion a formal charge of +2 is ascribed. The compound has diamagnetic properties.

The Mössbauer effect proved that in $\mathrm{C}_{60} \mathrm{Fe}_{2}$ the internal magnetic field on the Fe nuclei corresponds to one third of that for metallic iron [8].

It could be claimed that the magnetism of $\mathrm{C}_{60} \mathrm{Fe}_{x}$ samples originates from structural defects (e.g. iron grains). The linear increase in the saturation magnetization with doping, temperature dependence of the magnetic moment, and the Mössbauer effect measurements which revealed the sharp sextet pattern with h.f. field mentioned above, proved that this contribution is negligible. 


\section{Samples preparation and their crystalline structure}

The chromatographically purified $\mathrm{C}_{60}$ powder was impregnated with ferrocene and annealed in sealed ampoules for 72 hours at approximately $400^{\circ} \mathrm{C}$ (50 degrees below pure ferrocene decomposition temperature). It led to the decomposition of ferrocene to iron ions and remanent hydrocarbon groups, which condensed at the cold end of the ampoules in the form of white semitransparent crystallites (identified as mainly naphthalene). Next, the powder was annealed in vacuum to remove the residual volatile compounds.

The structure of the samples was checked by X-ray diffraction and high resolution electron microscopy. Neither of the techniques revealed iron or iron oxides or carbides grains. The crystalline structure of $\mathrm{C}_{60} \mathrm{Fe}_{x}$ resembles the structure of pure fulleride with the unit cell distorted by the increased distances between some of the $\mathrm{C}_{60}$ molecules (Fig. 1). Even for low iron concentration, the distor-

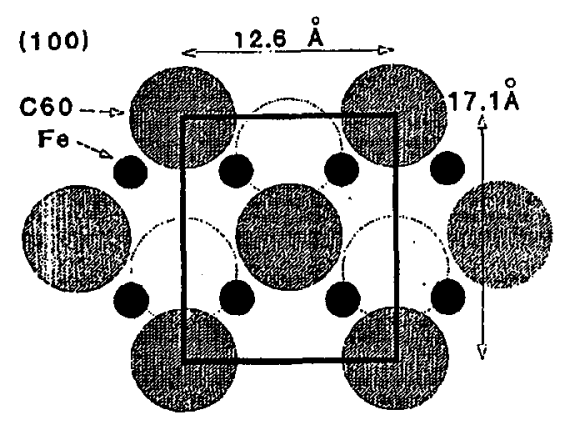

Fig. 1. The basal plane of the unit cell of the monoclinic $\mathrm{C}_{60} \mathrm{Fe}_{2}$ compound.

tion leads to new reflexes in the X-ray diffraction pattern. In the lattice, the iron atoms occupy the sites between two $\mathrm{C}_{60}$ molecules, and each molecule has up to four Fe nearest neighbors (Fig. 1). All four Fe atoms belong to the same (111) plane which is distorted in one of the three equivalent directions. The distortion of the (111) plane propagates in one direction only. With the stacking sequence ABCABC of the (001) planes of the new structure, it is best described as monoclinic with $a=24.3 \AA, b=12.6 \AA, c=17.1 \AA$, and $\beta=96.9$ with the $(a, b)$ basal plane constructed of the (111) plane of the pristine fulleride. For low concentration of iron in the crystal lattice, not all of the accessible sites are occupied. Nevertheless, the formation of $\mathrm{C}_{60} \mathrm{FeC}_{60} \mathrm{FeC}_{60} \mathrm{FeC}_{60} \ldots$ chains is probably responsible for the long range order. The proposed structure explains the limited solubility of the iron in $\mathrm{C}_{60}$ crystal lattice. The maximum of iron concentration, obtained with the applied doping method, corresponds to the composition $\mathrm{C}_{60} \mathrm{Fe}_{2}$.

The doping with iron considerably modifies the IR absorption spectrum [9] in comparison with pure fullerides (e.g. [10]). There appear several new absorption wide bands, as well as splitting of the molecular lines, which may originate from the molecules distortion. The main differences in the IR absorption, ascribed to the molecules vibration, were seen on both sides of the $T_{1 \mathrm{u}}(4)\left(1430 \mathrm{~cm}^{-1}\right)$ pentagon 
pinching mode (as new bands at 1461 and $1377 \mathrm{~cm}^{-1}$ ) and asymmetric broadening of the $T_{1 \mathrm{u}}(1)\left(527 \mathrm{~cm}^{-1}\right)$ breathing mode (at low temperatures, on low energy side of this mode a side band appeared at $\left.522 \mathrm{~cm}^{-1}\right)$. The modification of the $T_{1 \mathrm{u}}(1)$ mode supports the proposed structure of the molecule rigidly bound by $\mathrm{Fe}$ ions around its equator to its nearest neighbors. It should have different vibration frequencies of the in- and out- of the equator direction (or: the degeneracy of the mode is removed).

\section{Magnetic properties}

The magnetic measurements both at room temperature and at $5 \mathrm{~K}$ revealed the behavior typical of weak ferromagnet. The measurements of temperature dependence of magnetization were performed both in zero field cooling (ZFC) and field cooling (FC) regimes. The obtained results indicate significant role of the thermal history of samples in magnetic ordering processes. The magnetization being always positive, in the case of ZFC samples was usually lower than for the FC samples. The both curves converge, at some experimental conditions, as high as at $230 \mathrm{~K}$ in dependence on the thermal history of the samples. The magnetization of ZFC samples does not vary monotonically with the temperature but experiences the step-like increase at about 20 and $120 \mathrm{~K}$. The magnetic moment

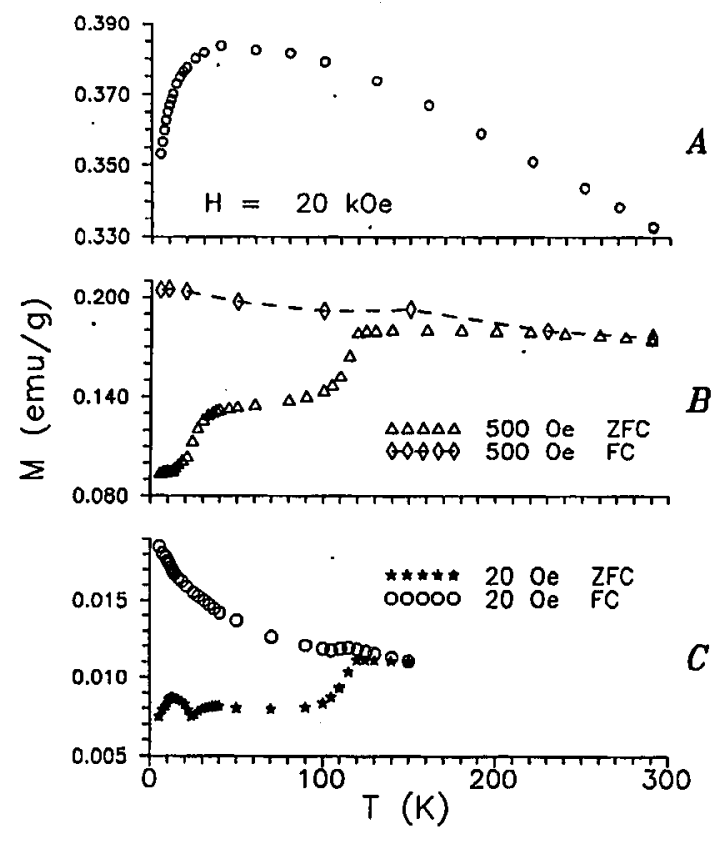

Fig. 2. The temperature dependence of magnetization of $\mathrm{C}_{60} \mathrm{Fe}_{0.5}$ for $\mathrm{ZFC}$ and $\mathrm{FC}$ sample in fields $20 \mathrm{Oe}$ and $500 \mathrm{Oe}$, and magnetization in $20 \mathrm{kOe}$ (corresponding to saturation state). 
for FC samples has tendency to increase with decreasing temperature and it varies steadily in comparison to that of ZFC samples. But even in magnetic field as high as $20 \mathrm{kOe}$, which well saturates the samples, and when no difference between ZFC and FC measurements were observed, the maximum of magnetization was found at about $40 \mathrm{~K}$. As an example, the temperature dependence of magnetization in magnetic fields $20 \mathrm{Oe}, 500 \mathrm{Oe}(\mathrm{ZFC}$ and FC) and in saturation state $(20 \mathrm{kOe})$ are presented in Fig. 2 for $\mathrm{C}_{60} \mathrm{Fe}_{0.5}$. The magnetic moment in saturation state at $40 \mathrm{~K}$ corresponds to about $0.1 \mu_{\mathrm{B}} / \mathrm{Fe}$.

\section{Discussion}

The iron ion in chemical compounds may be in either of the two ionization states as $\mathrm{Fe}^{2+}$ (in ${ }^{5} D_{4}$ state of a free ion) or $\mathrm{Fe}^{3+}$ (in ${ }^{6} S_{5 / 2}$ state). The ligand field of lower than octahedral symmetry removes the degeneracy of the ion ground state in both cases.

In this compound, according to the X-ray results, the iron bridges two molecules and it may be supposed, by comparison with ferrocene, that the position of $\mathrm{Fe}$ ions is determined by the interaction of individual pentagons from the nearest $\mathrm{C}_{60}$ molecules with $\mathrm{Fe}^{2+}$ ions. The ligand field is then of the fivefold symmetry with an inversion plane. Instead of the $d$ atomic orbitals, the hybridized atomic orbital $d^{3} p^{2}$ of fivefold symmetry should be used to analyze resonance valence bonds with the pentagons. If the energy levels structure of $\mathrm{C}_{60} \mathrm{FeC}_{60} \mathrm{com}$ plexes resembles that of ferrocene, then not all spins are paired. The incomplete spin pairing may be attributed to the imperfect alignment and orientation of the $\mathrm{C}_{60}$ pentagons which face the $\mathrm{Fe}^{2+}$ ions. The lattice vibration and the molecules librations modulate further the symmetry at the $\mathrm{Fe}^{2+}$ sites and the overlap of the ion atomic orbitals with $\mathrm{C}_{60} \pi$ electrons. At $20 \mathrm{~K}$ and $120 \mathrm{~K}$ it leads to sudden increase in the magnetic moment observed in the ZFC samples, to drastic changes in ESR linewidth and resonance field or in IR absorption to the $T_{1 \mathrm{u}}(1)$ mode splitting (measured above $25 \mathrm{~K}$ ). Apparently, at these temperatures there occurs the reconfiguration of the $\mathrm{C}_{60} \mathrm{FeC}_{60}$ bonds induced by the lattice vibrations.

The external magnetic field applied prior to cooling (FC), even as low as $20 \mathrm{Oe}$, reorients the magnetic moments and the internal magnetic field covers the effect of bonds changes. Therefore, magnetization of the FC samples or their remanence vary smoothly during cooling down to $5 \mathrm{~K}$. The magnetic measurements prove that the magnetic complexes interact with each other. One of the interand intra-complexes coupling agents can be the Fe $4 s$ electrons which are ESR silent in the compounds with such high iron concentrations that each of the $\mathrm{C}_{60}$ belong to one or more of the $\mathrm{C}_{60} \mathrm{FeC}_{60}$. The other competing magnetic interaction mechanism results from the de/formation of the valence bonds between cation and pentagons on both adjacent molecules. The bonds formation dictates the spin order in the $\mathrm{C}_{60} \pi$ electrons within each of the bounded molecules and further via the next iron ion.

The orientation of the magnetic moment induced by external magnetic field can be frozen in the samples if they are cooled in magnetic field and be observed as the remanence magnetization. Similarly, the ESR resonance field also depends 
on the cooling method. The onset of freezing takes place at approximately $230 \mathrm{~K}$ and it seems that the frozen state is reached at $20 \mathrm{~K}$. The thermal irreversibility, observed both in ESR and magnetization measurements, and the localized magnetic moments larger than found from the saturation magnetization points to the competition of ferro- and antiferromagnetic interactions in the $\mathrm{C}_{60} \mathrm{Fe}_{x}$ compounds and to their behavior similar to spin glasses with the spin correlation length extending beyond the second nearest neighbor. For low $x$ each complex $\mathrm{C}_{60} \mathrm{FeC}_{60}$ may be treated as weakly coupled by free electrons, with no direct $\pi$ electrons inter complex magnetic coupling. In case of high iron concentration, the whole two-dimensional spin network is being formed. In this model, magnetic moments are still confined to iron sites and total spin of the molecules $\pi$ electrons is compensated.

The experimental procedure did not allow to distinguish any diamagnetic contribution to the measured effects. Although the $\mathrm{C}_{60}$ molecular orbitals scheme was used throughout the text, the discussed valence bonds limit the applicability of the scheme.

\section{Conclusions}

The magnetic properties of the $\mathrm{C}_{60} \mathrm{Fe}_{x}$ compound have to be described in terms of the localized interacting magnetic moments. Earlier, in [7] where ESR measurements were presented, these interactions were not taken into account in discussion, since those experiments did not supply sufficient information on the magnetic behavior of the compounds.

The observed properties entitle us to suggest that the compound could be considered as the spin glass. In the proposed model the Fe spin interact via the $\mathrm{C}_{60}$ $\pi$ electrons due to the valence bonding in the complex $\mathrm{C}_{60} \mathrm{FeC}_{60}$. The interaction requires transient polarization of $\mathrm{C}_{60} \pi$ electrons. The $\mathrm{Fe} 4 s$ electrons in the $\mathrm{C}_{60} t_{1 \mathrm{u}}$ derived molecular orbital probably also participate in the magnetic coupling of the molecules.

This work was partly supported by the Committee for Scientific Research under grant No. P302 25703.

\section{References}

[1] R.F. Curl, R.E. Smalley, Science 242, 1017 (1988).

[2] R.C. Haddon, A.F. Hebard, M.J. Rosseinsky, D.W. Murphy, S.C. Duclos, K.B. Lyons, B. Miller, J.M. Rosamilia, R.M. Fleming, A.R. Kortan, S.H. Glarum, A.V. Makhija, A.J. Muller, R.H. Eick, S.M. Zaharuk, R. Tycko, G. Dabbagh, F.A. Thiel, Nature 350, 320 (1991).

[3] A.F. Hebard, M.J. Rosseinsky, R.C. Haddon, D.W. Murphy, S.H. Glarum, T.T.M. Palstra, A.P. Ramirez, A.R. Kortan, Nature 350, 600 (1991).

[4] A.R. Kortan, N. Kopylev, S. Glarum, E.M. Gyorgy, A.P. Ramirez, R.M. Fleming, O. Zhou, F.A. Thiel, P.L. Trevor, R.C. Haddon, Nature 352, 230 (1992).

[5] A.R. Kortan, N. Kopylov, S. Glarum, E.M. Gyorgy, A.P. Ramirez, R.M. Fleming, F.A. Thiel, R.C. Haddon, Nature 355, 529 (1991). 
[6] P.M. Allemand, K.C. Khemani, A. Koch, F. Wudl, K. Holczer, S. Donovan, G. Gruner, J.D. Thompson, Science 253, 301 (1991).

[7] P. Byszewski, R. Jablonski, J. Piechota, Proc. RAMIS'93, Poznain (Poland) 1993, in press.

[8] D. Satula, private communication.

[9] P. Byszewski, K. Antonova, R. Diduszko, M. Baran, R. Jablonski, to be published.

[10] W. Kraetschmer, K. Festiropoulus, D.R. Huffman, Chem. Phys. Lett. 170, 167 (1990). 